\title{
Molecular dynamics simulations of nanoporous organosilicate glasses using reactive force field (ReaxFF)
}

\author{
J.M. Rimsza, Lu Deng, and Jincheng Du* \\ ${ }^{l}$ Department of Materials Science and Engineering, University of North Texas, Denton, Texas, \\ U.S.A. \\ (*Corresponding author. Email: du@unt.edu)
}

\begin{abstract}
The structure and properties of nanoporous organosilicate glass (OSG) structure models with 30-70\% porosities and different pore morphologies were simulated using molecular dynamics simulations with Reactive Force Field (ReaxFF) potential. The OSG structures were created from nanoporous silica structures generated using classical molecular dynamics (MD) simulations with partial charge pairwise potentials and a subsequent step of adding hydroxide and methyl groups to the dangling bonds and coordination defects. The nanoporous OSG systems were then fully relaxed using molecular dynamics simulations with ReaxFF and detailed structure analysis performed and properties calculated. Analysis of the OSG systems indicated that the structural features (bond distances and angles) as well as the $\mathrm{Q}_{\mathrm{n}}$ distribution of the nanoporous silica backbone structure are consistent with the features of dense silica and that the geometry of the added methyl groups are experimentally accurate, even after ReaxFF relaxation. The elastic modulus of the nanoporous silica was calculated and found to be 24-31 GPa for system with $30 \%$ porosity and $0.5-2.5 \mathrm{GPa}$ for those with $70 \%$ porosity, consistent with previously reported experimental results.
\end{abstract}

\section{Introduction}

Porous silica is of significant interest due to the variability and tunability of its pore structures ranging from the molecular to the nanometer and the micrometer scale, which enables their applications in sensors, catalysis, hydrogen storage, drug delivery, thermal insulation, microelectronics, and photonics [1-3]. Organosilicates glasses (OSG) are nanoporous silica systems that are functionalized by introducing organic content including methyl groups and hydrocarbon chains which, together with the porosity, significantly lowers the dielectric content from 3.9 (amorphous silica) to 2.2-2.8 (OSG) and finds applications as low-k dielectrics in VLSIC (Very Large Scale Integrated Circuit) microelectronic devices [4]. The variability of nanoporous silica and OSG systems (order/disordered structures, large/small pores, high/low 
organic content, and pore morphologies) makes them well suited to a wide range of applications but there is a lack of reliable information concerning their structure-property relationships.

As a result, atomistic simulations have been applied to understand their microstructures and its relationship to mechanical and other properties. The understanding of OSG is especially limited due to their complex organic-inorganic hybrid bonding and different pore morphologies. This paper addresses these challenges by combining molecular dynamic (MD) simulation protocols to create nanoporous silica structures using MD with ReaxFF relaxation to generate realistic OSG structure models.

The complexity of OSG systems (which is further compounded by the randomized porosity) can make them difficult to characterize by computational or experimental methods. For dense silica experimental methods including NMR (nuclear magnetic resonance) and electron diffraction have been effective in providing some insight into the coordination environments, bond lengths, and bond angles [5]. The addition of light organic elements to the nanoporous silica to form OSG generates an increasingly complex system which has undergone limited experimental analysis, with the expectation of FTIR which has provide information on various bond concentrations $[6,7]$.

Attempts have been made to standardize the description of porosity through the analysis of the fractal geometry, absorption isotherms, and relative elastic modulus $\left(\mathrm{E} / \mathrm{E}_{\mathrm{o}}\right)$ and porosity $(\mathrm{P})$ relationships of the systems [8-11]. Due to the ease of calculating the elastic modulus of experimental and computational systems the relationship between the pore structure and the elastic modulus has been extensively studied by Jain et al, Roberts et al, and Rice, among others, who have demonstrated that it is possible to describe the type of porosity depending on the $\mathrm{E} / \mathrm{E}_{\mathrm{o}}$ v. P trend [12-14]. Porosity is usually described by fitting the $\mathrm{E}_{\mathrm{E}} \mathrm{E}_{\mathrm{o}} \mathrm{V}$. P relationship with either a power or an exponential fit, through which the value of the coefficient is analyzed [12-14].

Development of nanoporous silica models, used as the basis for OSG structure generation, have focused on creating well-ordered porosity (such as perfect spherical pores) which have not fully captured the variability of experimental porosity [15-18]. Two exceptions include work by Roberts and Garboczi using the finite element method (FEM) and Rimsza and Du using classical MD to investigate the role of different porosity regimes on changes in the mechanical properties of nanoporous silica systems $[13,19]$. Investigation of porosity regimes resulted in the 
description of randomized porosity as "stacked spherical particles" or "stacked spherical pores" which provides insight into the base structure of the porosity [13,19].

Development of realistic OSG structure models has been met with several hurdles related to the complex structure of the systems. The first is the amorphous nature of nanoporous silica backbone, followed by the randomization of the porosity, and finally the existence of organic groups. The amorphous backbone structure indicates that it is desirable to create large simulations (several thousand atoms) so that statistical analysis of the systems can take place. The size of the system limits the application of more computationally accurate but expensive calculation methods to OSG development including density functional theory (DFT) based methods. While several classical MD forcefields have been developed to create computational silica models (including those by Van Beest, Kramer, and Santen (BKS), Hassanali and Singer, Muralidharan and colleagues, and Feuston and Garofalini) none of these take into account the existence of either porosity or organic groups [20-23]. Therefore, it is necessary to implement a multistep approach to create the OSG computational structure models for further analysis.

Another difficulty of atomistic simulations of OSG is the organic-inorganic hybrid nature of the system which generates complex and varied bonding states in the system. The mixed bonding character of these systems pose significant challenges of force fields for atomistic simulations of these materials. More accurate first principles methods, such as DFT, can be employed but they significantly limiting the system size that can be studied due to high computational cost [24]. The recent development of Reactive Force Field (ReaxFF) enabled the description of these complex systems with high fidelity. A previous study of the reaction between poly-dimethylsiloxane (-O$\left.\mathrm{Si}\left(\mathrm{CH}_{3}\right)_{2-}\right)$ and $\mathrm{H}_{2} \mathrm{O} / \mathrm{SiO}_{2}$ demonstrates that ReaxFF can produce the thermal properties and time evolution of products at various temperatures and pressures realistically [25]. The successful application of ReaxFF to this combined silica and hydrocarbon systems clearly demonstrates the advantages of using ReaxFF for complex systems with mixed bonding states.

In this paper a series of OSG structure models were created by combining charge scaling and lattice expansion MD simulations with effective partial charge potentials to generate nanoporous amorphous silica with subsequent addition of organic methyl groups and eventual full relaxation of the structures using MD with ReaxFF. ReaxFF allows for the development of large scale structure models which include the randomized porosity and organic content critical to the 
microstructure of nanoporous silica and OSG while still generating experimentally accurate structures.

The article is arranged as follows: first a description of the classical MD and ReaxFF methods used in this work, then the development of the initial nanoporous silica models from two different classical MD protocols, the generation of OSG structure models, analysis of the atomic structure and the mechanical properties, followed by discussions and conclusions.

\section{Methods and simulation details}

\subsection{MD simulation procedure}

In this work the initial 3000 atom model nanoporous silica systems were developed using the parallel MD simulation package DL_POLY 2.2 with cubic periodic boundary conditions [26]. A set of partial charge pairwise potentials were used which included a long-range columbic interaction with a short-range interaction in the Buckingham form which has been successfully applied to silicate glass simulations by Du and Cormack [27,28]. Berendsen thermostats and barostats were used to control the temperatures and pressure in the canonical (NVT) and isothermal-isobaric (NPT) ensembles used to create the randomized porosity in the simulations. Additional details concerning the classical MD simulations methods can be found in reference [19]. All simulations were performed on the Talon and Eagle supercomputing clusters at the University of North Texas.

\subsection{Reactive Force Field (ReaxFF)}

ReaxFF, developed by Adri van Duin and coworkers, is a force field based on atomic bond order which allows for the computation of bond formation and disassociation, as well as the modeling of chemical reactions $[29,30]$. The ability to describe bond formation and bond dissociation arises from the relationship between bond distance and bond order/energy which ReaxFF employs. The calculation scheme of geometry-dependent charge (EEM scheme) is applied to calculate the atomic charges in the computational systems [31]. In ReaxFF the energy of the system is calculated through the use of nine different energy terms in order to consider the various types of interatomic interactions which can occur[29,30]. The equation for the energy of the system is included below [29, 30]:

$$
E_{\text {sys }}=E_{\text {bond }}+E_{\text {over }}+E_{\text {under }}+E_{\text {val }}+E_{\text {pen }}+E_{\text {tors }}+E_{\text {conj }}+E_{\text {vdWaals }}+E_{\text {Coulomb }}
$$


$\mathrm{E}_{\text {bond }}$ is the energy which is generated from the atoms bonding state [29]. $\mathrm{E}_{\text {over }}$ is an energy penalty for over-coordination of the atom [29]. $E_{\text {under }}$ is an energy contribution from the $\pi$ electron when it is unbonded[29]. $\mathrm{E}_{\mathrm{val}}$ is a valence angle term which approaches zero as bond disassociation occurs, ensuring that there is not a sharp transition during bond breakage and formation [29]. $E_{p e n}$ is an energy penalty which is included to account for the possible occurrence

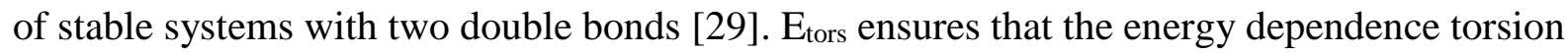
angle smoothly approaches zero during bond disassociation [29]. $\mathrm{E}_{\mathrm{conj}}$ accounts for conjugate effects in the molecular energy [29]. $E_{\mathrm{vdWaals}}$ is a long range valence interaction which is accounted for on all atoms and uses a distance corrected Morse potential [29]. Finally, $\mathrm{E}_{\text {Coulomb }}$ is a columbic interaction which is applied to all atomic pairs [29]. ReaxFF is particularly well equipped to account for bond breakage and formation in complex systems which are too large to be treated with DFT methods due to the focus on maintaining a gradual decrease in the bond energy as atoms move apart, rather than experiencing a stepwise energetic change at a set energy boundary. For further descriptions and functional forms of the nine energy terms which are described above the reader is directed to the original paper in references [29,30].

\subsection{Nanoporous silica structure generation: lattice expansion (LE) and charge scaling (CS) methods}

Numerous methods of generating nanoporous silica computational models have been proposed in literature including the random removal of silica tetrahedron from dense silica models, the generation of perfectly stacked spherical pores, and randomized mixtures of two phases models with the removal of a sacrificial phase [13,16,17,32-35].

In this work two methods of generating randomized pore structure were used to mimic the complex experimental structures of sol-gel and CVD (chemical vapor deposition) derived nanoporous silica by implementation of the lattice expansion and charge scaling methods, respectively [19,36-38]. The lattice expansion (LE) method mimics a CVD derived experimental nanoporous silica structure and was described by Kieffer and Angell and further developed by Nakano et al [37,38]. The LE method begins with dense amorphous silica systems produced from a melt and quench method described in pervious papers [27]. After the perfect silica structure has been generated the system undergoes a stepwise linear expansion in all three dimensions by $20 \%$. The $20 \%$ expansion results in all the bonds stretching to create a looselydebonded structure. The expansion step was followed by annealing using classical NVT 
(constant number of atoms, volume and temperature) MD simulations for 30ps at 300K which allows the $\mathrm{Si}-\mathrm{O}$ bonds to reform, resulting in porosity distributed throughout the system. The expansion and relaxation steps were repeated to create nanoporous silica systems with porosities ranging from $30 \%$ to $70 \%$.

The charge scaling (CS) method generates structures which mimic those created from sol-gel experimental methods and was originally developed by Beckers and de Leeuw [36]. The CS protocol begins with a low-density random starting structure (densities between 0.21-1.21 $\mathrm{g} / \mathrm{cm}^{3}$ ) with initial ionic charges decreased to $20 \%$ of the initial values [36]. The decreased atomic charges allows for atomic movement and the generation of a homogenous initial mixture without the implementation of high temperature simulations [36]. The system was then thermalized using NVT conditions at $300 \mathrm{~K}$. The ionic charges were increased at rates of 5-15\% for every 20ps of simulation under a NVT ensemble. The amplification of the ionic charges causes increased interactions between the atoms until a nanoporous silica structure is formed. Once the ionic charges are at their final values the systems are thermalized for 30ps to create equilibrium porous structures.

\subsection{Nanoporous and organosilicate structure generation}

Development of realistic nanoporous and OSG systems used the above nanoporous silica models as frameworks for the creation of OSG systems. Nanoporous silica models contain several defect species which are not seen in experimental systems. These include non-bridging oxygen's (NBO), where an oxygen atom is bound to one silicon leaving the other bond dangling, under bonded silica, $\left(\mathrm{Si}^{3}\right)$ where a silicon atom is bonded to three oxygen instead of four, and two-membered rings, where two $\mathrm{SiO}_{4}$ tetrahedron are edge rather than corner sharing. To generate the OSG structure the existence of the computational defects are exploited to both remove the defects as well as add organic content to the system. Initially, two-membered ring defects are identified by the existence of a strained O-Si-O bond of less than $105^{\circ}$ and a bridging oxygen is removed, forming a set of $\mathrm{Si}^{3}$. Then, all the $\mathrm{Si}^{3}$ defects are identified in the system and are terminated with a methyl $\left(\mathrm{CH}_{3}\right)$. The addition of the $\mathrm{CH}_{3}$ generates $\mathrm{Si}-\mathrm{C}$ bonds and creates the organic content inside the system. Finally, all of the NBOs are terminated with $\mathrm{H}$ generating silanols (Si-OH). Once all defects have been terminated the OSG is relaxed using ReaxFF forcefield by employing alternating minimization and short NVE routines to allow for a slow and 
methodical system relaxation. The ReaxFF simulations were performed using USER-REAXC package in MD simulation package LAMMPS [39-41]. An example of the relaxed OSG structure is included in Figure 1.a. with a close-up of the added methyl groups in Figure 1.b. The location of the organic groups, on the internal surface areas of a nanoporous silica matrix, is consistent with previous work by Tajima and coworkers who developed OSG structure models using a nanoporous silica backbone structure with additional $\mathrm{Si}-\mathrm{OH}$ and $\mathrm{Si}-\mathrm{CH}_{3}$ groups [24].

a).

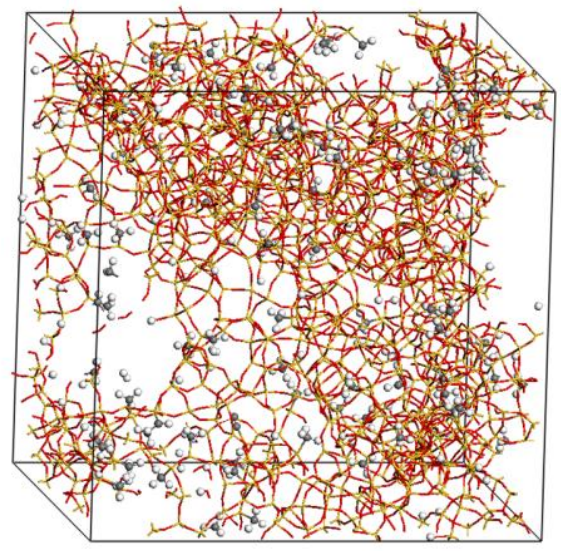

b)

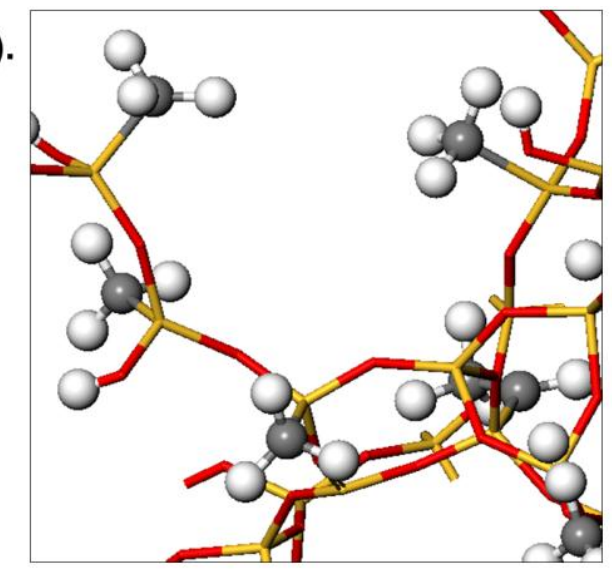

Figure 1: $53.5 \%$ porous organosilicate structure models (a) 3000 atom system (b) close-up of $\mathrm{CH}_{3}$ placement. Silica backbone structure is red (oxygen) and yellow (silicon) with the added hydrogens (white) and carbon (gray) atoms

\subsection{Structure analysis}

Structure analysis was performed on the relaxed OSG systems to analyze the accuracy of the nanoporous silica backbone structure and the geometry of the added methyl groups after ReaxFF relaxation. The structural analysis includes calculation of pair distribution functions (PDF) which can identify the primary bond distances as well as bond angle analysis (BAD) through which both the connectivity ( $\mathrm{Si}-\mathrm{O}-\mathrm{Si}$ bond angles) and regularity (O-Si-O bond angles) of the systems can be identified. BAD and PDF analyses were average over 30000 configurations recorded every 50 steps during ReaxFF NVE simulations. $Q_{n}$ distributions, which are used to describe the connectivity of amorphous systems, where also calculated.

Mechanical properties of the nanoporous silica backbone structures were calculated to provide another benchmark of validating differences in the two types of nanoporous silica systems. An energy minimization method implemented in the GULP code was used to obtain the stiffness and compliance matrices from which the isotropic elastic modulus is calculated using the Voight-Reuss-Hill (VHR) formalism [27]. 


\section{Results}

\subsection{Atomic structure of organosilicate glasses}

\subsubsection{Simulated structures with different porosities}

Porous nanoporous silica systems were generated using the lattice expansion (LE) and charge scaling (CS) method at approximately $30 \%, 50 \%$ and $70 \%$ porosity and then converted into OSG structures. Perfect control of the porosities of the nanoporous silica backbone structures was not possible due to allowing for volumetric changes to remove any negative pressure regions arising from the introduction of porosity, though variations in porosity are less than 5\%. Snapshots of the simulated nanoporous OSG systems are including in Figures 2 and 3 at the three different porosity levels.
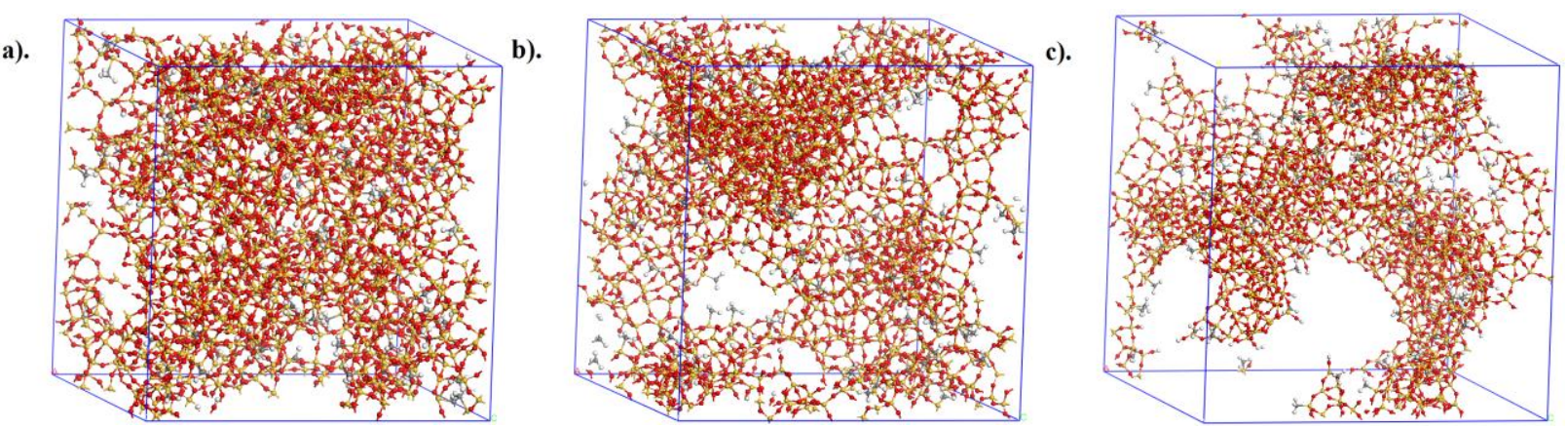

Figure 2: Simulated nanoporous OSG structures using ReaxFF for the charge scaling (CS) porosity method at a) $30.85 \%$, b) $50.65 \%$, and c) $70.23 \%$ porosity. The red is the oxygen atoms and the yellow is silicon.
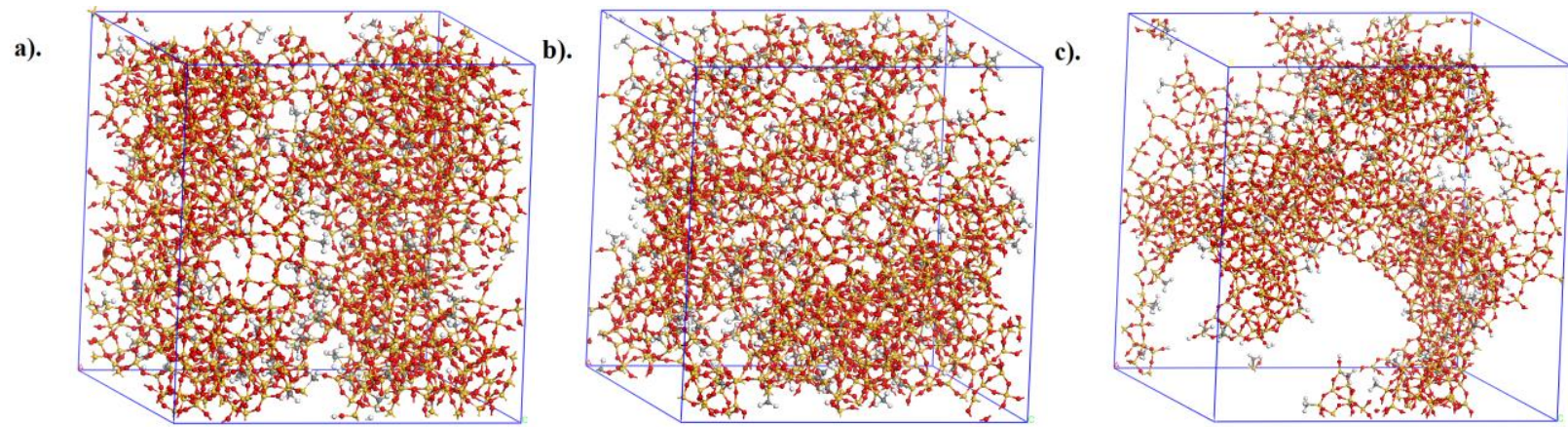

Figure 3: Simulated nanoporous OSG structures using ReaxFF for the lattice expansion (LE) porosity method at a) $32.94 \%$, b) $46.29 \%$, and c) $73.08 \%$ porosity. The red is the oxygen atoms and the yellow is silicon.

\subsubsection{Pair distribution functions (PDF) analysis}


The pair distribution functions (PDF) of the organosilicate systems were analyzed to investigate the average $\mathrm{Si}-\mathrm{O}, \mathrm{O}-\mathrm{O}$, and $\mathrm{Si}-\mathrm{Si}$ bond distances in the nanoporous silica backbone structure while $\mathrm{Si}-\mathrm{C}$ and $\mathrm{C}-\mathrm{H}$ bond distances were used to examine the structure of the methyl.

The Si-O PDF, shown in Figure 4.a., exhibits a primary peak at 1.57-1.59 $\AA$ which is in agreement with previous simulation work as well as neutron/x-ray diffraction and ${ }^{17} \mathrm{O}$ dynamic angle spinning (DAS) NMR data $[5,19,38,42,43]$. There are no significant changes in the average Si-O distance with changing porosity or the method of developing the nanoporous silica backbone structure, suggesting that the introduction of internal surface area does not affect the Si-O bond length. The Si-Si PDF has a primary peak at $3.05 \AA$ (Figure 4.b.) which is similar to the experimental value of $3.05 \AA$ with a standard deviation of $0.067 \AA$ which was derived from ${ }^{17} \mathrm{O}$ DAS NMR data [5,44]. Again, there appears to be little change in the Si-Si PDF with porosity or simulation method indicating that there is little difference in the nanoporous silica backbone of the OSG system. The O-O PDF plot indicates that the O-O distance is around 2.52 $\AA$ (Figure 4.c.) which is slightly shorter than the $2.65 \AA$ reported through the use of $\mathrm{x}$-ray diffraction and the range of 2.58-2.67 from XAFS (x-ray absorption fine structure) methods [43][45]. The slight difference between the computational values presented here and previous experimental studies by Mozzi and Warren and Cheah et al may be the result of a slightly higher concentration of three-membered rings in the computational structure compared to the experimental systems which can cause oxygen atoms to be in closer proximity to one another $[43,45]$. 
a).

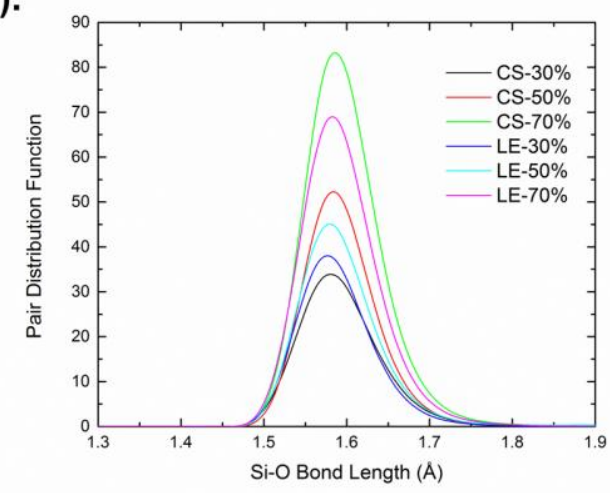

b).

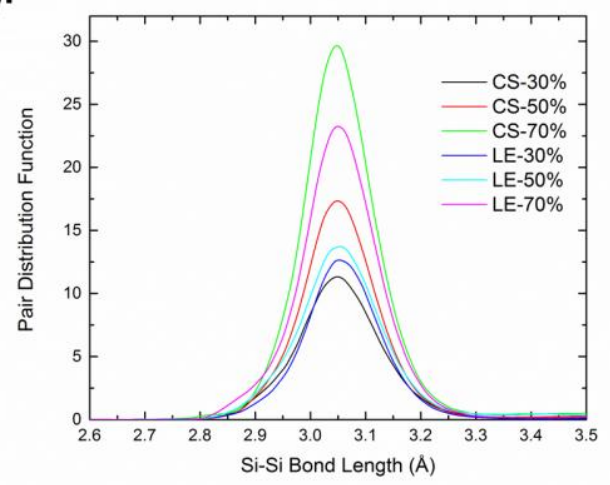

c).

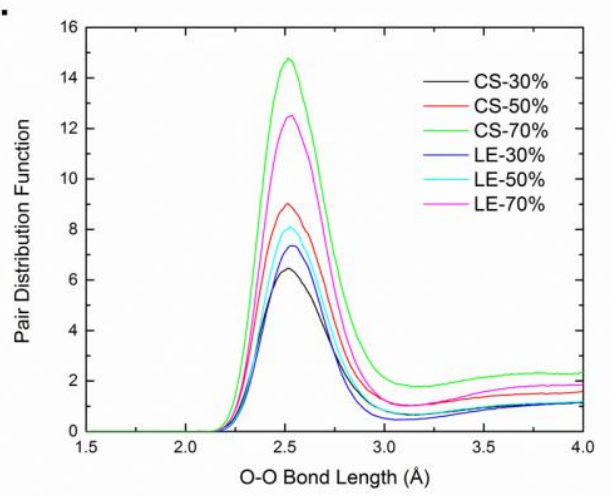

Figure 4: Pair distribution function of the silica backbone structure in nanoporous OSG a) Si-O PDF primary peak b) Si-Si PDF primary peak c) O-O PDF primary peak

The Si-C PDF has a primary peak at $1.97 \AA$ (Figure 5.a.), which is $\sim 0.1 \AA$ longer than the $1.875 \pm 0.002 \AA \mathrm{Si}-\mathrm{C}$ bond lengths in hexamethydisilane $\left(\mathrm{Si}_{2}\left(\mathrm{CH}_{3}\right)_{6}\right)$ which was measured by electron diffraction [46]. A similar Si-C bond length of $1.865 \pm 0.004 \AA$ was found in the $\left(\mathrm{CH}_{3}\right)_{3} \mathrm{Si}-\mathrm{C} \equiv \mathrm{C}-\mathrm{H}$ gas phase molecule by electron diffraction [47]. The differences between the computational $\mathrm{Si}-\mathrm{C}$ values reported here and the experimental value of $1.97 \AA$ maybe the result the relative weakness of the $\mathrm{Si}-\mathrm{CH}_{3}$ bond compared to the $\mathrm{Si}-\mathrm{O}$ bonds which are present, in contrast to the hexamethydisilane where each $\mathrm{Si}$ is bonded to three methyl groups and the neighboring silicon atom [46]. Previous DFT studies on TMCTS (trimethylcylictrisiloxane) molecule identified a $\mathrm{Si}-\mathrm{CH}_{3}$ bond length of $1.89 \AA$ when the $\mathrm{Si}$ coordination environment contains two bridging oxygen, one hydrogen, and a methyl group, demonstrating a slight $\mathrm{Si}-\mathrm{C}$ bond lengthening with an increase in the number of bridging oxygen [6]. Furthermore, the bulk silica surface may cause lengthening of the $\mathrm{Si}_{-} \mathrm{CH}_{3}$ bond and may factor in the slightly longer $\mathrm{Si}$ - 
$\mathrm{CH}_{3}$ bond length. Even so, the bond length is between 0.08-0.1 $\AA$ larger than what is expected experimentally, an error of only $4-5 \%$, which can be considered a reasonable deviation. The C-H PDF exhibits a peak at $1.12 \AA$ (Figure 5.b.) which is consistent with the length of $1.127 \pm 0.009 \AA$ reported for $\mathrm{C}-\mathrm{H}$ bonds in $\mathrm{Si}-\mathrm{CH}_{3}$ structures measured by electron diffraction in hexamethyldisilane[46]. The consistency of the $\mathrm{C}-\mathrm{H}$ bonds demonstrates that the added methyl groups and related $\mathrm{Si}-\mathrm{CH}_{\mathrm{x}}$ groups which were developed during the ReaxFF relaxation are consistent with bond lengths reported by experimental results[46].

a).

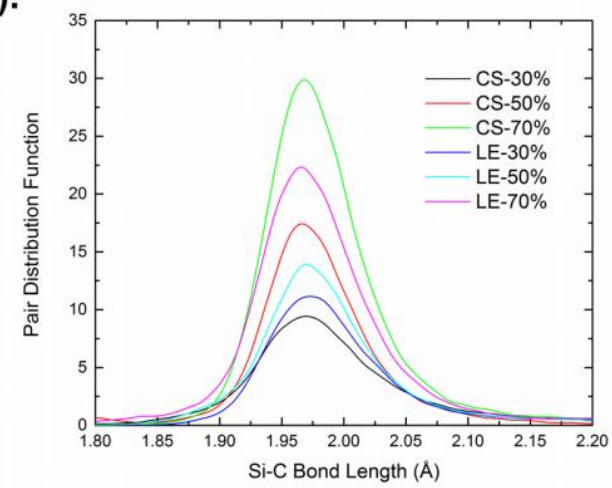

b).

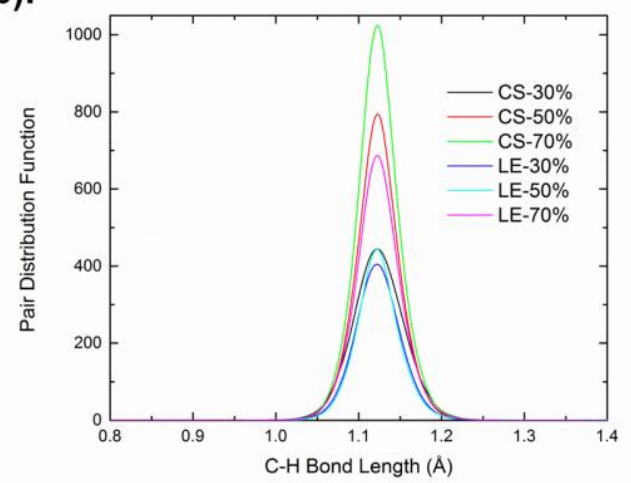

Figure 5: Pair distribution function of silanol and methyl groups in nanoporous OSG a) Si-C PDF primary peak b) C-H PDF primary peak

\subsubsection{Bond angle distribution functions (BAD) analysis}

The analysis of the bond angle distribution (BAD) of the nanoporous OSG systems describes the medium range order characteristics of the computational models. The BAD plot of $\mathrm{Si}-\mathrm{O}-\mathrm{Si}$ bond angle indicates that the main peak is located around $145^{\circ}$ (Figure 6.a.). Si-O-Si bond angles for experimentally derived silica vary from $130^{\circ}-180^{\circ}$ with the most common peak values being reported as between $147^{\circ}-151^{\circ}$ [5]. Variation in the $\mathrm{Si}-\mathrm{O}-\mathrm{Si} \mathrm{BAD}$ peak can be accounted for by differences in experimental methods, high energy x-ray and neutron diffraction studies which measure the $\mathrm{Si}-\mathrm{O}$ bond distances from which the $\mathrm{Si}-\mathrm{O}-\mathrm{Si}$ bond angles are calculated, and MAS (magic angle spinning) NMR, which uses the quadrupoler asymmetry parameter to predict the Si-O-Si angle [5]. Additionally, the value of the Si-O-Si bond angle is known to vary depending on the ring size distribution of the system. Silicon atoms which are in two-membered ring defects exhibit a highly strained Si-O-Si bond angle of $98^{\circ}$, resulting in a high energy configuration which only occur experimentally after fracturing of silica rods in a vacuum $[19,48]$. The Si-O-Si bond angles of $126.7^{\circ} \pm 4.1^{\circ}, 142.3^{\circ} \pm 10.9^{\circ}$, and $147^{\circ} \pm 12.2^{\circ}$ for 
three-membered, four-membered, and five+ membered rings respectively as measured by ${ }^{29} \mathrm{Si}$ DAS NMR $[5,49]$. Therefore, the average Si-O-Si bond angle of $145^{\circ}$ reported in this work is consistent with previous computational results for systems which have an average ring size between 4-5 which is expected for dense silica as well as with x-ray diffraction data reported by Mozzi and Warren $[19,43]$. Previous work by Rimsza and Du used similar methods of generating nanoporous silica structures and found that nanoporous silica structures tend to have a slightly lower average ring size at $\sim 6$ compared to $\sim 7$ for bulk silica, accounting for some of the differences in the Si-O-Si BAD primary peak [19]. The slight variation between the BAD for the $30 \%$ and $50 \%$ LE OSG systems may arise from a slightly different ring size distribution between the systems, since the lower the porosity the more similar the structure is too dense silica, suggesting that there is a slightly higher concentration of five and six membered rings. Experimentally, lower average ring concentrations will occur in silicate glasses with a low fictive temperature, which generates smaller ring structures and a lower Si-O-Si bond angle[5]. The lack of a pre-peak at $\sim 100^{\circ}$ indicates that the high energy two-membered ring defects, which generate localized strain on the silica surface and shorter Si-O-Si bond angles, have been successfully removed and were not reformed during ReaxFF relaxation [19].

a).

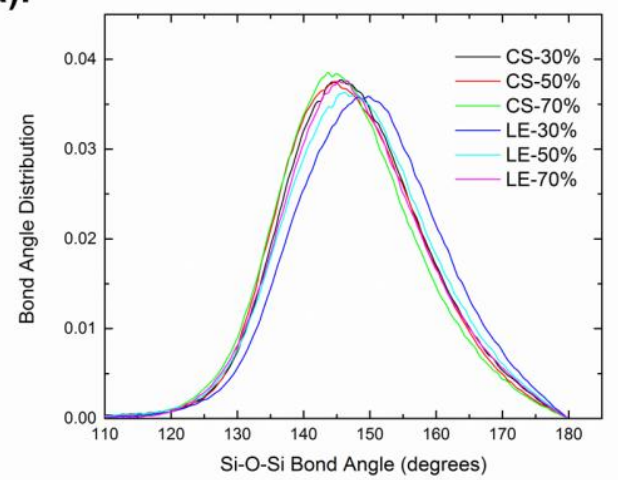

b).

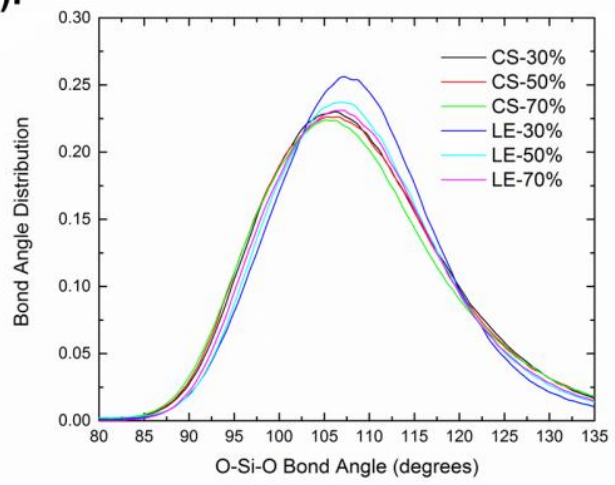

Figure 6: Bond angle distribution of the silica backbone structure in nanoporous OSG a) Si-O-Si BAD primary peak and b) O-Si-O BAD primary peak

The differences levels of variation in the Si-O-Si and O-Si-O BAD are due to the flexibility of the Si-O-S angle, which, due to its larger range, is capable of contracting and expanding, causing differences in the Si-O-Si peak value. For instance, in previous work Rimsza and Du noted that during the generation of nanoporous silica running a pressure controlled simulation resulted in a $9^{\circ}$ decrease in the $\mathrm{Si}-\mathrm{O}-\mathrm{Si} \mathrm{BAD}$ primary peak [19]. In contrast the O-Si- 
$\mathrm{O}$ bond angle is controlled by the formation of a $\mathrm{SiO}_{4}$ tetrahedron, which typically does not experience significant variation. The primary peak of O-Si-O BAD plot is centered at $107^{\circ}$ (Figure 6.b.) which is similar to the $109.5^{\circ}$ which is expected for the perfect silicon tetrahedral and the range of $109.3^{\circ}-109.7^{\circ}$ which has been experimentally derived through the use of neutron and high-energy x-ray diffraction [28]. The slight deviation in the O-Si-O BAD primary peak value for the computational models seen here may be accounted for by the density of three membered rings in the nanoporous silica backbone which can generate slightly constrained O-SiO BAD values of $95^{\circ}-105^{\circ}$. Due to the lack of a peak or a shoulder in the O-Si-O BAD at $\sim 100^{\circ}$ there is no significant distortion in the nanoporous silica structure, such as may arise from the existence of two-membered rings, other than a slight underestimation of the $\mathrm{O}-\mathrm{Si}-\mathrm{O}$ bond angle. The analysis of the PDF and BAD data of the nanoporous silica models relaxed with ReaxFF indicates that the nanoporous silica backbone structure is consistent with bond angles and lengths which were previously reported for experimentally derived silica by neutron and x-ray diffraction as well as MAS NMR experiments [5, 28, 43, 49].

The Si-C-H and H-C-H BAD values can provide insight into the structure of the added $\mathrm{Si}-\mathrm{CH}_{\mathrm{x}}$ groups which were introduced into the OSG structure. The Si-C-H BAD has a primary peak located at $108^{\circ}$ (Figure 7.a.) which is similar to the values of $109.2^{\circ} \pm 0.8^{\circ}$ and $108.7^{\circ} \pm 0.8^{\circ}$ which was reported by electron diffraction of tetramethylsilane and hexamethylsilane respectively [46]. Similarly the H-C-H BAD has a primary peak between $111^{\circ}-112^{\circ}$ (Figure 7.b.) which is consistent with values of $110.3 \pm 0.8^{\circ}$ from the same experiment [46]. The consistency between the Si-C-H and H-C-H BAD primary peaks with experimental data indicates that there is no distortion of the methyl groups due to the use of ReaxFF to relax the system.

a).

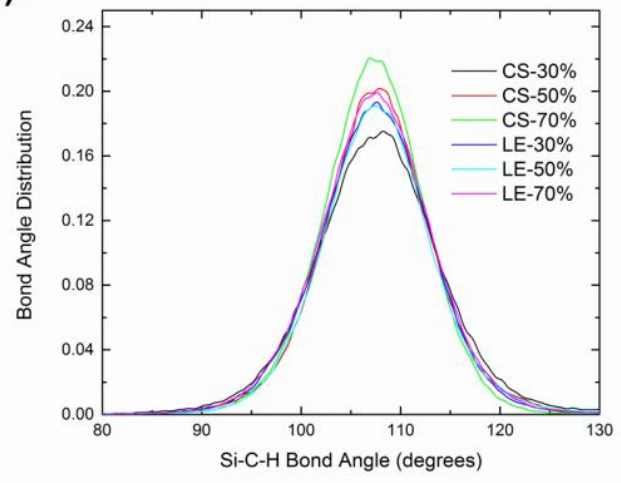

b).

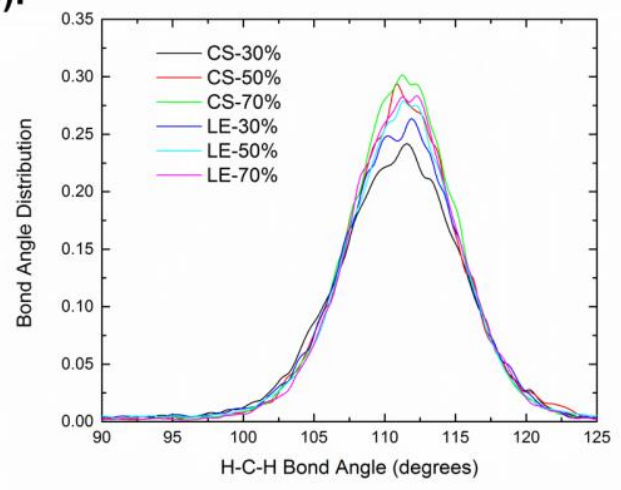


Figure 7: Bond angle distribution of silanol and methyl groups in nanoporous OSG a) Si-C-H BAD primary peak and b) $\mathrm{H}-\mathrm{C}-\mathrm{H}$ BAD primary peak

\subsubsection{Qn distribution}

The analysis of the defect concentrations and $\mathrm{Q}_{\mathrm{n}}$ distributions in the nanoporous OSG models is critical since it describes the regularity of the structure and can have a significant effect on the macroscopic properties of the system, including the mechanical strength. The $Q_{n}$ distributions describe the proportion of the network formers ( $\mathrm{Si}$ ) in the glass that are connected to bridging oxygen. A perfectly bonded $\mathrm{Si}$ atom in an amorphous network would have a $\mathrm{Q}_{4}$ value of 100 , indicating that $100 \%$ of the Si are connected to four bridging oxygen. Due to the inclusion of porosity in the system it is expected that there will be a higher concentration of $\mathrm{Q}_{3}$ and $\mathrm{Q}_{2}$ species in the porous OSG than in dense silica. The LE derived OSG systems exhibit an increasing concentration of $\mathrm{Q}_{2}$ and $\mathrm{Q}_{3}$ species with increasing porosity, which results in a decreasing $\mathrm{Q}_{4}$ concentration as seen in Tables 1 and 2. Due to the development of increased porosity a higher proportion of -Si-O-Si- bonds are broken, generating the $\mathrm{Q}_{2}$ and $\mathrm{Q}_{3}$ species. The trend of increase $\mathrm{Q}_{2}$ and $\mathrm{Q}_{3}$ species with increasing porosity is not maintained for the CS derived OSG systems, with the $\mathrm{Q}_{4}$ distribution staying relatively consistent at $\sim 84 \%$ for the full porosity range (Table 2). The difference between the $\mathrm{Q}_{4}$ distribution trends is due to the development of the initial nanoporous silica structure. Since the development of the nanoporous silica backbone structure results in continual breakage of the $\mathrm{Si}-\mathrm{O}$ bonds during the expansion step, the number of $\mathrm{Q}_{4}$ species decreases linearly, since only a portion of the original connectivity is recovered. Meanwhile, in the CS developed nanoporous silica systems each of the systems are developed independently, leading to a more consistent molecular structure of the silica and the increased porosity being the result of added free space in the structure, rather than structural reorganization. A very low level of $\mathrm{Q}_{5}$ species are identified in OSG developed using both CS and LE simulation methods, with values decreasing with increasing porosity. A measureable concentration of $\mathrm{Q}_{5}$ species can occur in MD derived silica but typically in concentrations of less than $0.8 \%$, consistent with the very low levels of $Q_{5}$ species in the OSG systems[19,23,50]. $\mathrm{Q}_{\mathrm{n}}$ distribution analysis of $7 \mathrm{~nm}$ amorphous silica particles was performed by Kim and Lee using ${ }^{29}$ Si MAS NMR which identified $6 \pm 2 \% \mathrm{Q}_{2}, 34 \pm 5 \% \mathrm{Q}_{3}$, and $60 \pm 7 \% \mathrm{Q}_{4}$ [51]. The OSG systems studied here exhibit lower $\mathrm{Q}_{2}$ and $\mathrm{Q}_{3}$ distributions than what is reported by ${ }^{29} \mathrm{Si}$ MAS NMR[51]. The difference may be accounted for by the existence of a more ideal 
coordination of the silica backbone structure in the OSG systems compared to the amorphous silica particles. Kim and Lee noted that a portion of the $\mathrm{Q}_{2}$ and $\mathrm{Q}_{3}$ species exist not only on the surface but also inside the amorphous particles, a conclusion which was reached by comparison of the $\mathrm{Q}_{\mathrm{n}}$ distributions between $7 \mathrm{~nm}$ and $14 \mathrm{~nm}$ silica particles[51]. Computationally generated dense silica exhibits a $\mathrm{Q}_{\mathrm{n}}$ distribution with $0.0 \mathrm{Q}_{2}, 0.6 \mathrm{Q}_{3}, 99.3 \mathrm{Q}_{4}$, and $0.1 \mathrm{Q}_{5}$ species which supports the fact that the high $\mathrm{Q}_{2}$ and $\mathrm{Q}_{3}$ species present in this work is the result of the porosity $[28,52]$. Comparison of the $Q_{n}$ distribution pre ReaxFF (Table 1) and post ReaxFF relaxation (Table 2) demonstrate a slight increase in $\mathrm{Q}_{2}, \mathrm{Q}_{3}$, and $\mathrm{Q}_{5}$ species, usually less than $1 \%$ and an increase in the silanol concentration after ReaxFF relaxation. The deviation in $\mathrm{Q}_{\mathrm{n}}$ distribution maybe a result of the ability of ReaxFF to simulate more complex carbon containing environments, removing the energetic gain for generating a perfect silica structure, such as exists in dense silica MD forcefields. Ultimately, the $\mathrm{Q}_{\mathrm{n}}$ distribution demonstrates that the nanoporous silica backbone structure exhibits a higher $\mathrm{Q}_{2}$ and $\mathrm{Q}_{3}$ concentration than what is seen experimentally due to a higher connectivity in the backbone structure, with the concentration of $\mathrm{Q}_{2}$ and $\mathrm{Q}_{3}$ species arising predominantly from the development of internal surface area.

Table $1 \mathrm{Q}_{\mathrm{n}}$ distribution and silanol concentration of OSG structure models at $\sim 30 \%, \sim 50 \%$, and $\sim 70 \%$ porosity using the charge scaling (CS) and lattice expansion (LE) protocol before relaxation with ReaxFF.

\begin{tabular}{|c|c|c|c|c|c|c|c|c|c|c|}
\hline & \multicolumn{2}{|c|}{$\mathrm{Q}_{2}$} & \multicolumn{2}{|c|}{$\mathrm{Q}_{3}$} & \multicolumn{2}{|c|}{$\mathrm{Q}_{4}$} & \multicolumn{2}{|c|}{$\mathrm{Q}_{5}$} & \multicolumn{2}{|c|}{ Silanol } \\
\hline & CS & LE & CS & LE & $\mathrm{CS}$ & $\mathrm{LE}$ & CS & LE & $\mathrm{CS}$ & LE \\
\hline $30 \%$ & 0.8 & 2.3 & 13.7 & 15.4 & 85.5 & 82.0 & 0.0 & 0.3 & 2.43 & 3.27 \\
\hline $50 \%$ & 1.4 & 2.2 & 13.4 & 18.3 & 85.2 & 79.4 & 0.0 & 0.1 & 2.81 & 3.04 \\
\hline $70 \%$ & 2.2 & 2.2 & 16.6 & 18.8 & 81.1 & 79.0 & 0.1 & 0.0 & 3.80 & 3.53 \\
\hline Dense & \multicolumn{2}{|c|}{0.0} & \multicolumn{2}{|c|}{0.6} & \multicolumn{2}{|c|}{99.3} & \multicolumn{2}{|c|}{0.1} & \multicolumn{2}{|c|}{0.1 (NBO) } \\
\hline
\end{tabular}

Table $2 \mathrm{Q}_{\mathrm{n}}$ distribution and silanol concentration of OSG structure models at $\sim 30 \%, \sim 50 \%$, and $\sim 70 \%$ porosity using the charge scaling (CS) and lattice expansion (LE) protocol after relaxation with ReaxFF.

\begin{tabular}{|c|c|c|c|c|c|c|c|c|c|c|}
\hline & \multicolumn{2}{|c|}{$\mathrm{Q}_{2}$} & \multicolumn{2}{c|}{$\mathrm{Q}_{3}$} & \multicolumn{2}{c|}{$\mathrm{Q}_{4}$} & \multicolumn{3}{c|}{$\mathrm{Q}_{5}$} & \multicolumn{2}{c|}{ Silanol } \\
\hline & $\mathrm{CS}$ & LE & CS & LE & CS & LE & CS & LE & CS & LE \\
\hline $30 \%$ & 1.1 & 2.4 & 14.1 & 15.4 & 84.1 & 81.7 & 0.7 & 0.5 & 2.58 & 3.27 \\
\hline $50 \%$ & 1.7 & 2.1 & 13.3 & 18.3 & 84.6 & 79.4 & 0.4 & 0.2 & 2.95 & 2.96 \\
\hline $70 \%$ & 2.5 & 2.6 & 16.8 & 18.9 & 80.4 & 78.5 & 0.3 & 0 & 3.87 & 3.82 \\
\hline Dense & \multicolumn{3}{|c|}{0.0} & \multicolumn{2}{|c|}{0.6} & \multicolumn{2}{c|}{99.4} & \multicolumn{2}{c|}{0.0} & $0.15(\mathrm{NBO})$ \\
\hline
\end{tabular}




\subsection{Porosity characterization and correlation to properties}

Concerns with the application of OSG as low-k dielectrics have centered on the degradation of the mechanical properties with increasing porosity. Specifically, low-k dielectric systems with elastic modulus (E) less than 10GPa cannot be successfully integrated into microelectronic processing $[4,53]$. The mechanical strength of the OSG systems is dictated by the strength of the nanoporous silica backbone structure, since the Si-O-Si linkages are significantly stronger than the $\mathrm{Si}_{-} \mathrm{CH}_{3}$ and $\mathrm{Si}-\mathrm{OH}$ surface bonds. It is the free volume which restricts the connectivity of the system, leading to significant decreases in the elastic modulus [54]. The OSG systems derived from LE methods experience significantly lower elastic modulus than the CS derived OSG over the porosity range, as seen in Figure 8. Iacopi et al found that organosilicate glass samples could be strengthened up to $40 \%$ through the application of UV light which results in an increase concentrations of Si-O-Si bridges and slightly smaller $\mathrm{Si}-\mathrm{O}-\mathrm{Si}$ bonds at $\sim 144^{\circ}$ [55]. From Table 2 it is clear that the LE derived OSG has consistently lower concentrations of $\mathrm{Q}_{4}$ species as well as higher concentrations of silanol groups (which were originally NBO defects until they were hydrogen terminated). Therefore, it is the loss of connectivity and increase in coordination defects which causes the lower mechanical properties, and is consistent with the previous reports by Iacopi et al [55]. The mechanical property range of 5.5-14.2 for the LE and CS nanoporous silica backbone structure at 50\% porosity is consistent with the experimental range of 2.0-17 reported for experimental porous silica systems by nanoindentation [56-58]. The wide range of possible values for nanoporous silica systems arises form differences in deposition method, drying times, and the structure of porosity [56-58]. Nanoporous silica systems from both simulation methods demonstrated $\mathrm{E} / \mathrm{E}_{\mathrm{o}}-\mathrm{P}$ trends which can be fit with power or exponential functions [19]. Previous analysis of nanoporous silica systems by Rimsza and Du indicated that they are similar to experimentally derived systems generated from CVD or sol-gel processing based on the value of the exponent in the power or exponential fit [19]. 


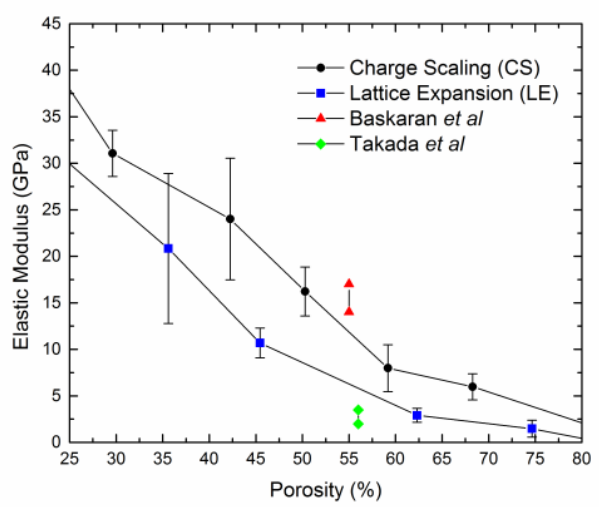

Figure 8: Elastic modulus of nanoporous silica systems generated using the charge scaling (CS) or lattice scaling (LE) protocol as well as comparison with experimental nanoporous silica data from nanoindentation $[56,57]$. The error bars for the computational data are the standard deviation of three simulation sets.

\section{Discussion}

The OSG structure is consistent with what is expected with the nanoporous silica backbone structure based on the reported bond angles and lengths for experimental dense silica and the structure of the methyl groups being comparable to what is reported by experiment using NMR and x-ray/electron diffraction[28,46,51]. The calculated mechanical properties are also well within the range of 2.0-17 GPa reported for nanoporous silica system from nanoindentation data. For the OSG systems, while the structure is consistent with experiment, the organic content is low, between 2.68-5.05 atomic \% though the location of the methyl groups, on the interior surfaces, is consistent with other computational models of OSG [24]. OSG low-k dielectrics obtained from PECVD method usually have higher organic content between 29-46 mole \% for systems with dielectric constants between 2.5-3.0, which suggests that additional organic content is needed in addition to the introduction of methyl groups. Higher organic content can be achieved by introducing hydrocarbon chain or partially replacing surface silicon to carbon in the nanoporous silica structures [59]. The inclusion of additional carbon based groups in the OSG is expected to be easily accommodated by ReaxFF, since even with the current OSG systems some more complex hydrocarbon groups were formed during the relaxation of adjacent methyl groups. Additionally, based on the success of the application of ReaxFF to mixed silanol and hydrocarbon systems it may also be possible to generate a full porous OSG systems from a protocol similar to the two presented here [25]. 


\section{Conclusions}

Realistic organosilicate (OSG) model systems with porosities ranging from $30 \%$ to $70 \%$ were created by combining fixed partial charge potentials and ReaxFF molecular dynamics (MD) simulations. The protocol included the generation of a nanoporous silica structure with randomized porosity to which hydroxide or methyl/hydrocarbon groups were added to terminate the dangling bonds creating the nanoporous silica or OSG structures. Analysis of the backbone structure demonstrates that the systems exhibit both short and medium range structural features of amorphous silica that is consistent with experimental results. Additionally, mechanical properties of these structure models were calculated and it was found that the elastic moduli is dependent on the concentration of Si-O-Si linkages, which can be correlated with an increase silanol percentages and decreasing $\mathrm{Q}_{4}$ species. Future work includes increasing the organic content of the OSG systems as well as investigating how the added organic groups affect mechanical behaviors and water diffusion.

\section{Acknowledgments}

Funding support for this work is provided by DOE NEUP project (\#13-5494) and SRC Task \#2071.024. J.M. Rimsza acknowledges funding from the NSF Graduate Research Fellowship Program. We gratefully acknowledge helpful discussions with Prof. Adri van Duin. 


\section{Reference List}

[1] J.J. Li, X.Y. Xu, Z. Jiang, Z.P. Hao, C. Hu, Nanoporous silica-supported nanometric palladium: Synthesis, characterization, and catalytic deep oxidation of benzene, Environ. Sci. Technol. 39 (2005) 1319-1323.

[2] I. Fisher, W. Kaplan, M. Eizenberg, Dielectric property-microstructure relationship for nanoporous silica based thin films, J. Appl. Phys. 95 (2004) 5762-5767.

[3] R. Morris, P. Wheatley, Gas Storage in Nanoporous Materials, Angewandte Chemie International Edition. 47 (2008) 4966-4981.

[4] D. Shamiryan, T. Abell, F. Iacopi, K. Maex, Low- $<\mathrm{i}>\mathrm{k}</ \mathrm{i}>$ dielectric materials, Materials Today. 7 (2004) 34-39.

[5] T.M. Clark, P.J. Grandinetti, P. Florian, J.F. Stebbins, Correlated structural distributions in silica glass, Physical Review B. 70 (2004) 064202.

[6] H. Kazi, J.M. Rimsza, J. Du, J.A. Kelber, Ar ions and oxygen plasma interactions of amine terminated organosilicate glass (OSG): a combined experimental and ab intio simulation study, JVSTA. (2014).

[7] H. Kazi, J.A. Kelber, Plasma damage mechanisms in low k organosilicate glass and their inhibition by Ar ion bombardment, Journal of Vacuum Science \& Technology A. 32 (2013) 021302.

[8] D.W. Schaefer, K.D. Keefer, Structure of random porous materials: silica aerogel, Phys. Rev. Lett. 56 (1986) 2199.

[9] E.P. Barrett, L.G. Joyner, P.P. Halenda, The determination of pore volume and area distributions in porous substances. I. Computations from nitrogen isotherms, J. Am. Chem. Soc. 73 (1951) 373-380.

[10] R.W. Rice, The porosity dependence of physical properties of materials: a summary review, Key Eng Mat. 115 (1995) 1-20.

[11] R. Rice, Extension of the exponential porosity dependence of strength and elastic moduli, J Am Ceram Soc. 59 (2006) 536-537.

[12] A. Jain, S. Rogojevic, S. Ponoth, N. Agarwal, I. Matthew, W. Gill, P. Persans, M. Tomozawa, J. Plawsky, E. Simonyi, Porous silica materials as low- $<\mathrm{i}>\mathrm{k}</ \mathrm{i}>$ dielectrics for electronic and optical interconnects, Thin Solid Films. 398 (2001) 513-522.

[13] A.P. Roberts, E.J. Garboczi, Elastic properties of model porous ceramics, J Am Ceram Soc. 83 (2000) 3041-3048. 
[14] R.W. Rice, Evaluating porosity parameters for porosity-property relations, J Am Ceram Soc. 76 (2005) 1801-1808.

[15] H. Miyoshi, N. Hata, T. Kikkawa, Theoretical investigation into effects of pore size and pore position distributions on dielectric constant and elastic modulus of two-dimensional periodic porous silica films, Japanese journal of applied physics. 44 (2005) 1166.

[16] H. Miyoshi, H. Matsuo, Y. Oku, H. Tanaka, K. Yamada, N. Mikami, S. Takada, N. Hata, T. Kikkawa, Theoretical analysis of elastic modulus and dielectric constant for low-k twodimensional periodic porous silica films, Japanese journal of applied physics. 43 (2004) 498-503.

[17] H. Miyoshi, H. Matsuo, H. Tanaka, K. Yamada, Y. Oku, S. Takada, N. Hata, T. Kikkawa, Theoretical investigation of dielectric constant and elastic modulus of two-dimensional periodic porous silica films with elliptical cylindrical pores, Japanese journal of applied physics. 44 (2005) 1161.

[18] H. Miyoshi, K. Yamada, K. Kohmura, N. Fujii, H. Matsuo, H. Tanaka, Y. Oku, Y. Seino, N. Hata, T. Kikkawa, Theoretical investigation of dielectric constant and elastic modulus of threedimensional isotropic porous silica films with cubic and disordered pore arrangements, Japanese journal of applied physics. 44 (2005) 5982.

[19] J. Rimsza, J. Du, Structural and Mechanical Properties of Nanoporous Silica, J Am Ceram Soc. 97 (2014) 772-781.

[20] B. Van Beest, G. Kramer, R. Van Santen, Force fields for silicas and aluminophosphates based on ab initio calculations, Phys. Rev. Lett. 64 (1990) 1955.

[21] A.A. Hassanali, S.J. Singer, Model for the water-amorphous silica interface: The undissociated surface, The Journal of Physical Chemistry B. 111 (2007) 11181-11193.

[22] K. Muralidharan, J. Simmons, P. Deymier, K. Runge, Molecular dynamics studies of brittle fracture in vitreous silica: Review and recent progress, J. Non Cryst. Solids. 351 (2005) 15321542 .

[23] B. Feuston, S. Garofalini, Empirical three-body potential for vitreous silica, J. Chem. Phys. 89 (1988) 5818.

[24] N. Tajima, T. Ohno, T. Hamada, K. Yoneda, N. Kobayashi, S. Hasaka, M. Inoue, Molecular modeling of low-k films of carbon-doped silicon oxides for theoretical investigations of the mechanical and dielectric properties, Appl. Phys. Lett. 89 (2006) 061907-061907-3.

[25] K. Chenoweth, S. Cheung, A.C. Van Duin, W.A. Goddard, E.M. Kober, Simulations on the thermal decomposition of a poly (dimethylsiloxane) polymer using the ReaxFF reactive force field, J. Am. Chem. Soc. 127 (2005) 7192-7202. 
[26] T. Forester, W. Smith, The DL_POLY_2 Reference Manual, Daresbury Laboratory, Daresbury. (2000).

[27] J. Du, Molecular Dynamics Simulations of the Structure and Properties of Low Silica Yttrium Aluminosilicate Glasses, J Am Ceram Soc. 92 (2009) 87-95.

[28] J. Du, A.N. Cormack, Molecular dynamics simulation of the structure and hydroxylation of silica glass surfaces, J Am Ceram Soc. 88 (2005) 2532-2539.

[29] A.C. Van Duin, S. Dasgupta, F. Lorant, W.A. Goddard, ReaxFF: a reactive force field for hydrocarbons, The Journal of Physical Chemistry A. 105 (2001) 9396-9409.

[30] A.C. Van Duin, A. Strachan, S. Stewman, Q. Zhang, X. Xu, W.A. Goddard, ReaxFFSiO reactive force field for silicon and silicon oxide systems, The Journal of Physical Chemistry A. 107 (2003) 3803-3811.

[31] W.J. Mortier, S.K. Ghosh, S. Shankar, Electronegativity-equalization method for the calculation of atomic charges in molecules, J. Am. Chem. Soc. 108 (1986) 4315-4320.

[32] T. Coquil, J. Fang, L. Pilon, Molecular dynamics study of the thermal conductivity of amorphous nanoporous silica, Int. J. Heat Mass Transfer. 54 (2011) 4540-4548.

[33] A. Brodka, T. Zerda, Properties of liquid acetone in silica pores: Molecular dynamics simulation, J. Chem. Phys. 104 (1996) 6319-6326.

[34] L.D. Gelb, K. Gubbins, Characterization of porous glasses: simulation models, adsorption isotherms, and the Brunauer-Emmett-Teller analysis method, Langmuir. 14 (1998) 2097-2111.

[35] T.C. McDermott, T. Akter, J.M.D. MacElroy, D.A. Mooney, M.T.P. McCann, D.P. Dowling, Diffusion within Ultrathin, Dense Nanoporous Silica Films, Langmuir. 28 (2011) 506516.

[36] J. Beckers, S. De Leeuw, Molecular dynamics simulation of nanoporous silica, J. Non Cryst. Solids. 261 (2000) 87-100.

[37] J. Kieffer, C.A. Angell, Generation of fractal structures by negative pressure rupturing of $\mathrm{SiO}<\mathrm{sub}>2</$ sub $>$ glass, J. Non Cryst. Solids. 106 (1988) 336-342.

[38] A. Nakano, L. Bi, R.K. Kalia, P. Vashishta, Structural correlations in porous silica: Molecular dynamics simulation on a parallel computer, Phys. Rev. Lett. 71 (1993) 85-88.

[39] H.M. Aktulga, J.C. Fogarty, S.A. Pandit, A.Y. Grama, Parallel reactive molecular dynamics: Numerical methods and algorithmic techniques, Parallel Computing. 38 (2012) 245-259.

[40] S. Plimpton, LAMMPS user's manual, Sandia National Laboratory. (2005). 
[41] S. Plimpton, Fast parallel algorithms for short-range molecular dynamics, Journal of computational physics. 117 (1995) 1-19.

[42] A. Nakano, L. Bi, R.K. Kalia, P. Vashishta, Molecular-dynamics study of the structural correlation of porous silica with use of a parallel computer, Physical Review B. 49 (1994) 9441.

[43] R. Mozzi, B. Warren, The structure of vitreous silica, Journal of Applied Crystallography. 2 (1969) 164-172.

[44] Y.G. Gogotsi, I.V. Uvarova, Nanostructured Materials and Coatings for Biomedical and Sensor Applications, Springer, 2003.

[45] S. Cheah, G.E. Brown Jr, G.A. Parks, XAFS Spectroscopy Study of Cu (II) Sorption on Amorphous $\mathrm{SiO}<\mathrm{sub}>2</$ sub $>$ and $\gamma$ - $\mathrm{Al}<\mathrm{sub}>2</ \mathrm{sub}>\mathrm{O}<\mathrm{sub}>3</$ sub $>$ : Effect of Substrate and Time on Sorption Complexes, J. Colloid Interface Sci. 208 (1998) 110-128.

[46] B. Beagley, J. Monaghan, T. Hewitt, Electron-diffraction studies of tetramethylsilane and hexamethyldisilane, and discussion of the lengths of Si-C bonds, J. Mol. Struct. 8 (1971) 401411.

[47] W. Zeil, J. Haase, M. Dakkouri, Structure of molecules of type (CH 3) $3 \mathrm{X}-\mathrm{C}$ [triple bond, length as m-dash] C-Y (X [double bond, length as m-dash] Si, Ge, Y [double bond, length as m-dash] H, Cl) determined by electron diffraction, Discuss. Faraday Soc. 47 (1969) 149-156.

[48] A.S. D'Souza, C.G. Pantano, Hydroxylation and dehydroxylation behavior of silica glass fracture surfaces, J Am Ceram Soc. 85 (2002) 1499-1504.

[49] T. Charpentier, P. Kroll, F. Mauri, First-principles nuclear magnetic resonance structural analysis of vitreous silica, The Journal of Physical Chemistry C. 113 (2009) 7917-7929.

[50] B. Feuston, S. Garofalini, Topological and bonding defects in vitreous silica surfaces, J. Chem. Phys. 91 (1989) 564.

[51] H.N. Kim, S.K. Lee, Atomic structure and dehydration mechanism of amorphous silica: Insights from $29 \mathrm{Si}$ and $1 \mathrm{H}$ solid-state MAS NMR study of $\mathrm{SiO} 2$ nanoparticles, Geochim. Cosmochim. Acta. 120 (2013) 39-64.

[52] A. Tilocca, N.H. de Leeuw, A.N. Cormack, Shell-model molecular dynamics calculations of modified silicate glasses, Physical Review B. 73 (2006) 104209.

[53] M.R. Baklanov, K. Maex, M.R. Baklanov, K. Maex, Porous low dielectric constant materials for microelectronics, Philosophical Transactions of the Royal Society A: Mathematical, Physical and Engineering Sciences. 364 (2006) 201-215.

[54] S.J. Schneider, Engineering Materials Handbook: Ceramics and Glasses, ASM international, 1991. 
[55] F. Iacopi, Y. Travaly, B. Eyckens, C. Waldfried, T. Abell, E. Guyer, D. Gage, R. Dauskardt, T. Sajavaara, K. Houthoofd, Short-ranged structural rearrangement and enhancement of mechanical properties of organosilicate glasses induced by ultraviolet radiation, J. Appl. Phys. 99 (2006) 053511.

[56] S. Baskaran, J. Liu, K. Domansky, N. Kohler, X. Li, C. Coyle, G. Fryxell, S. Thevuthasan, R. Williford, Low dielectric constant mesoporous silica films through molecularly templated synthesis, Adv Mater. 12 (2000) 291-294.

[57] S. Takada, N. Hata, Y. Seino, N. Fujii, T. Kikkawa, Dependences of Young's modulus of porous silica low dielectric constant films on skeletal structure and porosity, J. Appl. Phys. 100 (2006) 123512-123512-5.

[58] C. Murray, C. Flannery, I. Streiter, S. Schulz, M. Baklanov, K. Mogilnikov, C. Himcinschi, M. Friedrich, D. Zahn, T. Gessner, Comparison of techniques to characterise the density, porosity and elastic modulus of porous low- $<\mathrm{i}>\mathrm{k}</ \mathrm{i}>\mathrm{SiO}<\mathrm{sub}>2</ \mathrm{sub}>$ xerogel films, Microelectronic engineering. 60 (2002) 133-141.

[59] B. Bittel, P. Lenahan, S. King, Ultraviolet radiation effects on paramagnetic defects in low-א dielectrics for ultralarge scale integrated circuit interconnects, Appl. Phys. Lett. 97 (2010) 063506-063506-3. 